\title{
WORKFORCE Description and development of a nurse-led cardiac assessment team
}

\author{
Authors: Chun Shing Kwok, ${ }^{\mathrm{A}}$ Tamara Naneishvili, ${ }^{\mathrm{B}}$ Sonia Curry, ${ }^{\mathrm{C}}$ Charlotte Aston, ${ }^{\mathrm{D}}$ Michelle Beeston, ${ }^{\mathrm{C}}$ \\ Sarah Chell, ${ }^{C}$ James Cripps, ${ }^{C}$ Bob Gunter, ${ }^{C}$ Debbie Jackson, ${ }^{C}$ Diane Thomas, ${ }^{C}$ Angela Jones, ${ }^{C}$ Helen Bethell, ${ }^{C}$ \\ Kully Sandhu, ${ }^{\mathrm{E}}$ Dot Morgan-Smith ${ }^{\mathrm{F}}$ and Rhys Beynon ${ }^{\mathrm{G}}$
}

A problem was identified where patient care was affected because of delays in receiving specialist cardiology input. This report describes the experience of developing a specialist cardiac assessment where senior cardiac nurses were trained to provide a 24 -hour presence in the emergency department (ED). We describe the service and our evaluation of the service. These dedicated specialised nurses can optimise patient management including admission or safely discharge patients with relevant follow-up when necessary. The team also runs three clinics per week with consultant support. The team of 10 nurses provides a cardiology opinion to approximately 400 patients a month in the ED and 100 patients a month in the acute medical unit (AMU). Eighty-seven per cent of patients are seen in the ED within 30 minutes of referral. Approximately $40 \%$ of patients reviewed are accepted directly into cardiology beds thus avoiding admission to the AMU. It has been estimated that 6 bed-days are saved each day, which translated to an estimated $£ 400,000$ each year. The team also provides outpatient rapid access services which generates $£ 121,792$ income for the directorate. We demonstrate that a cardiac nurse assessment team can provide a cost-effective 24 -hour presence in the ED.

KEYWORDS: Nursing, cardiac assessment team, cardiology

\section{Introduction}

Cardiovascular disease is a major problem in the UK, associated with significant morbidity and mortality which is a huge burden to healthcare services. ${ }^{1}$ Cardiology conditions account for a significant percentage of patients attending the emergency department (ED) and any medical take. In the UK, there were more than 1.6 million episodes related to cardiovascular disease in NHS

Authors: ${ }^{\text {A }}$ cardiology registrar, Royal Stoke University Hospital, Stoke-on-Trent, UK; ${ }^{\mathrm{B}}$ core medical trainee, Royal Stoke University

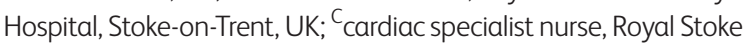
University Hospital, Stoke-on-Trent, UK; ${ }^{\text {D }}$ directorate manager, Royal

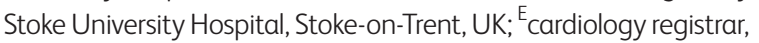
Royal Stoke University Hospital, Stoke-on-Trent, UK; F advanced nurse practitioner, Royal Stoke University Hospital, Stoke-on-Trent, UK; ${ }^{G}$ consultant cardiologist, Royal Stoke University Hospital, Stoke-onTrent, UK hospitals, accounting for $10 \%$ of all inpatient episodes among men and $6.2 \%$ among women. ${ }^{2}$ Pressures on hospital resources has led to a need for early specialist review so as to improve the patient journey and reduce unnecessary admissions.

Timely specialist input is critical in some cardiology conditions, such as acute myocardial infarction, acute heart failure and ventricular arrhythmias, and cardiology departments struggle to provide immediate specialist review due to lack of trained staff. Most district general hospitals (DGHs) do not have a dedicated cardiology registrar on call out of hours and in tertiary centres the on-call cardiology registrar may be busy in the catheter lab or managing acute emergencies. Therefore, there is great interest in developing innovative ways, such as a nurse-led service, to improve and provide more rapid cardiology specialist input.

A few previous studies have evaluated the role of nurses in early triage of patients. One study compared the management of non-ST elevation myocardial infarction patient prior and post development of a nurse-led early triage and found significant increases in electrocardiography performed within 10 minutes of admission, higher rate of prescription of clopidogrel and greater number of patients being managed in coronary care unit. ${ }^{3}$ There has also been an audit describing use of fast-track troponin measurements and specialist chest pain nurses which reduced time from chest pain onset to troponin blood test, reduced admissions to the hospital wards from the acute medical receiving unit, reduced admission time and reduced length of stay. ${ }^{4}$ This combination of fast-track measurements and specialist nurses saved approximately 2,000 bed-days per annum and released 5-6 acute beds per day. ${ }^{4}$ Specialist nurses have also been evaluated in triaging patients with syncope and have been found to have low predictive accuracy in identifying high-risk individuals. ${ }^{5}$ There has also been a qualitative study of nurses' cardiac triage decisions and they found that the nurse's ultimate goal is to have the patient receive prompt electrocardiography and prompt medical evaluation and to advocate for the patient. ${ }^{6}$ While nurses are aware of age and sex differences in myocardial infarction presentation, some nurses still hold cultural biases and stereotypes that may interfere with timely delivery of emergency cardiac healthcare. ${ }^{6}$

We describe our cardiology services before and after the development of the cardiac assessment nurse team. This description includes the specifics of the selection and training of the nurses and the services they provide. In addition, we describe our evaluation of the cardiac assessment nurse team service. 


\section{Cardiology services at our centre}

In addition to a coronary care unit and two cardiology wards, our cardiology service has four catheter labs (including a dedicated electrophysiology lab) and one minor procedure room for nurse-led loop recorder implants / direct current cardioversion. We also have a primary percutaneous coronary intervention (PCI) service and we are the largest pacing/device centre in UK and in the top six PCI (by volume) centres. We have a large structural heart disease programme in the catheter lab with transcatheter aortic valve implantation, Mitraclip, atrial septal defect closure and left atrial appendage occlusion procedures. Furthermore, we undertake a full spectrum of electrophysiology work and we have cardiothoracic surgery on site.

\section{Area for improvement in cardiology care}

In the UK, patients presenting to ambulance crews or EDs with acute ST elevation myocardial infarctions, complete heart block or ventricular tachycardia are usually directly referred to tertiary cardiac units or managed by DGH consultant cardiologists on call from home. These cases form a small portion of the patients presenting with potential cardiac conditions. The majority of patients are seen in the ED by ED doctors with three possible outcomes.

> Discharge from the ED without follow-up.

> Discharge from hospital and referred to cardiology outpatient services.

> Transfer to acute medical receiving units where patients are assessed on the post-take ward rounds. These patients are then often referred to cardiology units to be seen on the next cardiology ward round.

A group of cardiology consultants, senior nurses and managers concluded that this traditional model could be improved with the delivery of more timely care for patients at our tertiary centre. Patients would regularly spend up to 24 hours in hospital prior to being reviewed by the relevant specialty and patients were discharged home for outpatient appointments without any definitive assessment or investigation being arranged. Some patients were unnecessarily admitted with conditions that could be managed at home with the help of rapid access clinics. Significant patient dissatisfaction was noted with these delays and the local health economy perceived the pathway negatively due to the cost burden of additional outpatient appointments following ED presentation. Discussions with consultants from the ED and AMU revealed frustration with the level of cardiology input into the emergency portals. Like most hospitals in the UK, the Royal Stoke had significant bed pressures and outpatient appointment waits, and this traditional model did little to help reduce the length of stay and clinic backlogs. An example of patient flow prior to development of the cardiac specialist nurse team is shown for a patient presenting with chest pain (Fig. 1).

\section{Proposed solution to improve care: cardiac assessment nurse team service}

A working group of consultants, senior nurses and managers concluded that what was needed was a 24 hours a day senior cardiology presence in the ED. Ideally this would be provided by a stable workforce who would divert patients directly from the ED to the cardiology ward if appropriate, would assess and arrange appropriate outpatients investigation and follow-ups for patients who could be managed at home, and would reassure those patients who were not thought to have a significant cardiology condition.

It was clear to the department that the consultant body would not be able to provide this role, the on-call registrar was already too busy and we were unlikely to be able to attract enough registrars to provide a second cardiology on-call team. A potential solution was to intensively train a dedicated team of specialised senior cardiac nurses to be supported by the on-call registrar and consultants. A team of six would be required to provide a 24 -hour presence in the ED.

\section{Selection and training of the nurses}

The selection of the nurses involved a rigorous process. Applicants needed to have first level registration and at least 2 years
Fig. 1. Patient flow prior to the development of the cardiac specialist nurse team. $\mathrm{AMU}=$ acute medical unit; $\mathrm{ED}=$ emergency department; IP = inpatient; $\mathrm{OP}=$ outpatient; $\mathrm{SHO}=$ senior house officer.

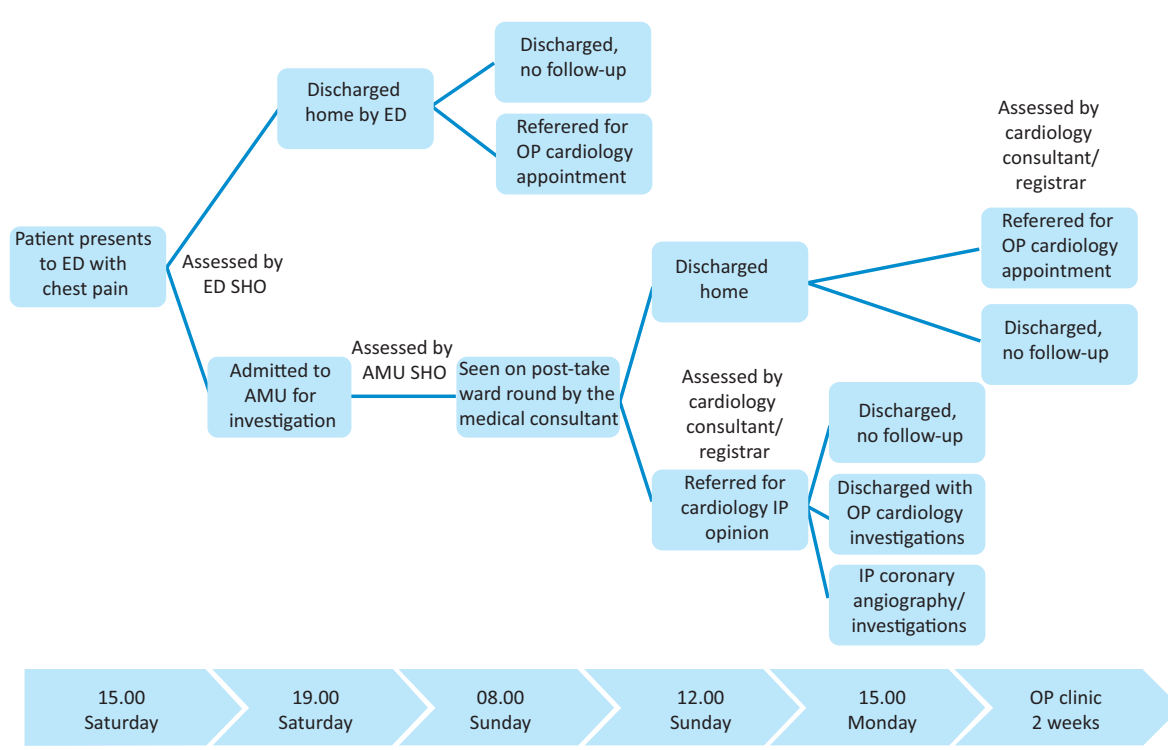


post-registration experience in cardiology as well as evidence of degree level study, excellent attendance and continued professional development. Desirable personality traits included confidence in decision making, proactive nature, willingness to take advanced levels of study and an understanding of the current NHS pressures.

The department successfully recruited six of its most senior nurses from the cardiology wards. Five of the six had at least 8 years cardiology experience and were ward sisters or charge nurses. Many had already undertaken a course in health assessment.

The successful applicants undertook a structured 'medical' training programme starting with an initial 9-12 month period of intensive supernumerary training involving both on job apprenticestyle training and formal academic study, all supervised by a consultant cardiologist acting as a mentor. During this time the nurses were paid as a band 5 nurse. All successful applicants agreed to a significant pay cut to join the team. Formal training included a health assessment module at master's level if not previously passed, followed by an 18-month postgraduate diploma in cardiology undertaken at Bradford University with 'in house' consultant mentoring throughout. This led to the qualification of 'practitioner with a special interest in cardiology'. The course included modules on heart failure, valvular disease, ischaemic heart disease, hypertension, arrhythmias and applied methodologies, including a clinical audit and service redesign module. Clinical objectives were set with consultant support and signed off by the consultant mentor. This qualification is recognised by the British Cardiovascular Society and a band 7 was awarded to nurses following completion of this postgraduate diploma. A full Master's in Cardiology was awarded upon completion of a dissertation module. Additional learning included non-medical prescribing, informal Ionising Radiation (Medical Exposure) Regulations training to enable X-ray requests and advanced life support qualifications. Overall, we aimed to achieve a qualified team with a broad medical cardiology knowledge combined together with the experience the nurses had gained previously on our acute wards. A rigorous process of in-house assessment also took place including clinical evaluation exercises (miniCEX) assessments, patient surveys and $360^{\circ}$ appraisals. The training programme is shown in Fig 2. The nurses were initially paid as a band 5 while training, and at time of the training they have no responsibilities and are supernumerary (not counted in the numbers). Once they have completed the health assessment and one of the Bradford modules, they are paid at band 6 and begin to operate in the role although they remain under supervision until the postgraduate cardiology diploma has been successfully completed.

The training programme was a considerable financial commitment for the trust at $£ 8,000$ per nurse covering the cardiology diploma and a prescribing course. A structured career plan was introduced that recognised milestones during the training programme with progression from band 5 to band 7 . Current costs for each band 7 staff is $£ 44,000$. The initial cost for the six-nurse team was $£ 264,000$. The initial team of six was determined to be the minimum requirement to provide the 24 hour presence in the ED without consideration of additional roles the cardiac assessment nurse team.

\section{Description of the cardiac assessment nurse service}

The service now provides timely specialist cardiology advice and support to emergency portals and the acute medical receiving

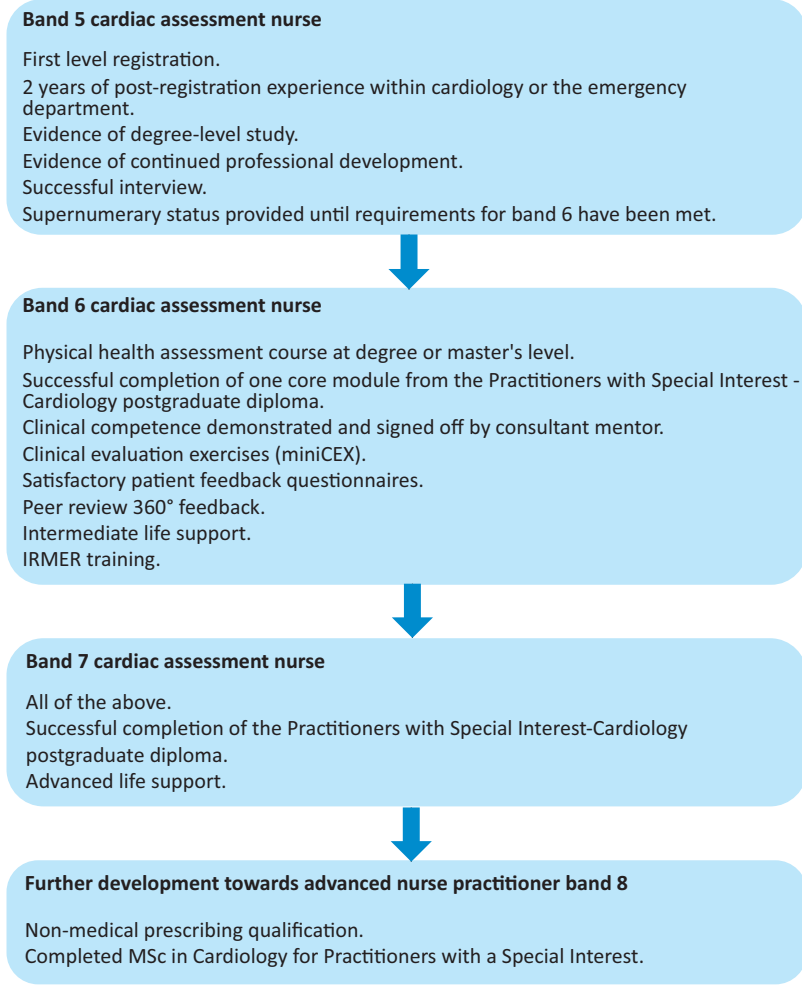

Fig. 2. Cardiac assessment nurse training programme. IRMER = Ionising Radiation (Medical Exposure) Regulations.

unit in our hospital with a large DGH population (approximately $500,000)$. Our trust stipulated that patients in the ED who were referred to specialist teams needed to be reviewed within 30 minutes of referral. The requirement of a 30 -minute review as an internal requirement of the trust's 'internal professional standards' which was initiated by the trust's executive team and agreed by all specialties in an effort to ensure the 4 -hour standard was addressed by the whole trust rather than just ED alone. The service also coordinates the primary PCI service.

The service now provides a cardiology opinion to 500 patients a month in the ED and AMU along with phone support to 200 ambulance crews, other departments in our trust, as well as advice to medical teams in local DGHs. The team provides a prompt assessment, a diagnosis and a management plan at the time of attendance. This can include direct admission to cardiology wards or arrangement for outpatient cardiac investigations and/or follow-up. The nurses hold the cardiology registrar bleep overnight diverting simple cases away from the registrar enabling the continuance of traditional on-calls and so maintaining daytime training opportunities. In addition, the cardiology assessment nurse team delivers both structured and informal training for ED doctors, nurses, and other healthcare professionals in the trust. The team run two rapid access chest pain clinics and one palpitations clinic per week with consultant support. These clinics provide 10 new patients slots for 50 weeks a year (rapid access chest pain) and four new and two follow-up slots in the palpitation clinic for 42 weeks a year. These clinics provide a revenue stream for the department and help the trust meet the 'referral to treatment' targets. Because of the additional roles, including 
informal training and rapid access clinics, the nursing team had to be expanded to a team of 10 .

At our centre, the cardiac assessment nurses carry the specialist registrar (SpR) bleep at night and there are two on-call consultants at any one time who were always happy to be contacted. At the beginning of the service, there was much consultation with the on-call cardiology SpR but this has declined as the service matured. During the inception of the model, differences in opinions were more of an issue but regular case-based meetings were pivotal in relationship and trust building between teams. Rarely, there were separate meetings with team leads to discuss any issues. In addition, the ED has recognised the transition of the role of the cardiac assessment nurses from being a 'signpost' to a 'decision maker' in the ED and this is a consequence of the commitment of a long-term investment from the cardiology consultant and management team.

An example of patient flow after the development of the cardiac specialist nurse team is shown for a patient presenting with chest pain (Fig 3).

Reflection is an important part of the cardiac assessment nurse service. Our team has six weekly consultant-led meetings where all members reflect on activities in order to try to improve services over time. During these meetings difficult cases are reviewed and team development is discussed. Speakers are invited from the consultant body and ideas shared among the team. Readmission rates, clinical management and breach rates are all assessed on a rolling audit programme with all patient interaction entered onto a team database. The team are managed by a lead nurse and a consultant clinical lead. There is day-to-day support from the wider cardiology consultant body.

The team is growing and now consists of 10 nurses. This has enabled the team to develop greater outreach work with interaction with the heart failure team in addition to support to the acute cardiology wards. Additional tasks performed by the specialist cardiac nurses include managing the rotas of the junior doctors and are working with them to facilitate earlier discharge of patients from the wards.

\section{Evaluation of the cardiac assessment nurse service}

The service now provides a cardiology opinion to approximately 400 patients a month in the ED and 100 patients a month in the AMU. Eighty-seven per cent of patients are seen in the ED within 30 minutes of referral. While the exact reason why $13 \%$ were not reviewed within 30 minutes of referral is not clear, it likely relates to either multiple referrals at the same time or one or more critical patients requiring imminent care so review of referrals had to be delayed. Approximately $40 \%$ of patients reviewed are accepted directly into a cardiology bed thus avoiding admission to the AMU. Ninety-one per cent of patients assessed by the team in ED, and then admitted to the AMU under general medicine, are discharged from hospital with a non-cardiac diagnosis. In terms of patient outcomes, $4 \%$ of the patients discharged from the ED were readmitted over a 6 -month period with a cardiac condition while $7 \%$ were readmitted for noncardiac reasons over the same period. This includes patients with recurrent supraventricular tachycardias and heart failure.

The cardiac assessment nurse team reduces costs by providing immediate cardiac opinions in the ED. This speeds up access to senior cardiology reviews, inpatient cardiac treatment and investigations such as coronary angiography as well. It has been estimated that 6 bed-days are saved each day which translated to an estimated $£ 400,000$ each year. There are additional cost savings from the rapid access chest pain and palpitation clinics which occur alongside consultant-led clinics. Considering the current national tariff for cardiology outpatient work is $£ 170$ for a new patient and $£ 98$ for follow-ups, this generates $£ 121,792$ income for the directorate.

The team has been extremely popular with both patients and hospital staff alike. Formal feedback from the ED consultant body and patient surveys have been repeatedly positive and individual $360^{\circ}$ appraisals for the team members have demonstrated a team with excellent clinical, organisational and interpersonal skills.

\section{Discussion}

We have described our innovative nurse-led cardiology assessment service that has improved standards and care for patients who present to our trust with acute cardiology conditions. We have shown that this is an effective service that can reduce time to specialist opinion, length of stay and costs to the health service. We believe that at a time of spiralling demand in the NHS, our Stoke cardiac assessment unit offers a solution that may be successfully rolled out to other trusts.

The literature suggests that specialist cardiac nurses are involved in different aspects of care to improve patient care and cardiology
Fig. 3. Patient flow after the development of the cardiac specialist nurse team. $\mathrm{ED}=$ emergency department; $\mathrm{IP}=$ inpatient; $\mathrm{OP}=$ outpatient.

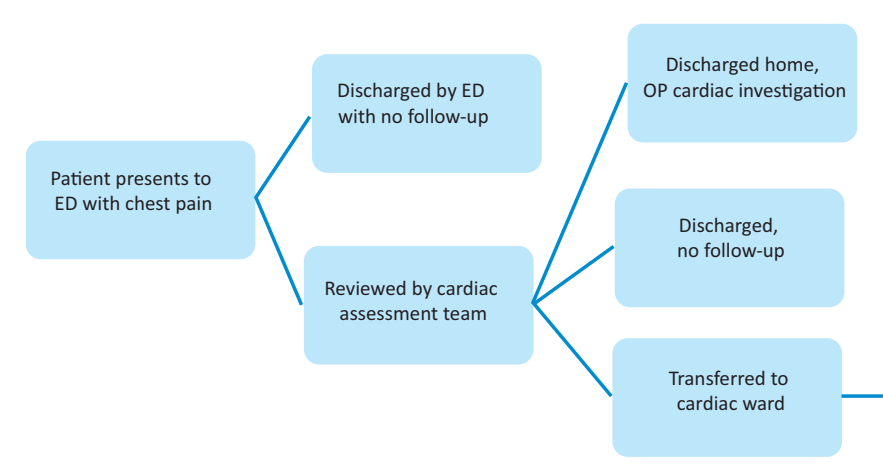

Cardiology post-take ward round and IP coronary angiography/investigation 
services, for example, in the management of chronic heart failure, a randomised trial found that nursing intervention with planned home visits and telephone contact was associated with reduced length of hospital stay and death or readmission. ${ }^{7}$ Other studies have shown that an intervention with specialist nurse working with other members of the multidisciplinary team can reduce adverse events within 6 months and increase survival free of readmission. ${ }^{8,9}$ In addition, another study compared care from a cardiac acute care nurse practitioner and medical team compared to medical team alone and found that nurse practitioner care was associated with reduced readmissions at 30 days. ${ }^{10}$ There is also evidence that cardiac specialist nurses can successfully run clinics where rapid assessments and evaluation of patients can be made for patients with suspected chest pain, heart failure or cardiac arrhythmia. ${ }^{11}$ There is limited literature regarding the role of cardiac specialist nurse teams providing early assessment of patients in the ED.

Patient care has improved as a result of this new service. First, there is a team which can review patients within 30 minutes of referral, thus reducing delays to cardiology services. Second, patients benefit from better management because of the first specialist review is from a static team of staff that is highly trained and experienced which accommodates prompt clinical decision making and direct access to SpR/consultant advice when needed, thus avoiding the impact of delays to patient care. Third, there is improved care as a consequence of the continued learning environment fostered by the team and performance reviews are regularly undertaken to ensure evidence-based practices are followed with support for team members in terms of continued development and opportunities to keep up with continuing professional development. Finally, the satisfaction among the team is high which improves staff morale which reduces burnout, so high-quality patient care can be delivered.

There were some challenges and important considerations associated with implementing the cardiology specialist nurse team. Initially, the team of nurse specialists found the prospect of providing expert opinions to ED consultants daunting and the team were significantly dependent on the cardiology registrar. There were also concerns in the ED regarding the team's experience and knowledge. The team's management decided that it was vital that the nurses undertook a formal prolonged course in cardiology rather than a typical advanced nurse practitioner (ANP) type master's course. The Bradford 'Cardiology for Practitioners with a Special Interest' master's course based in Bradford provided this. Some of the nurses were concerned about starting further academic study but all embraced the course and found it immensely rewarding and confidence building. Over the course of 12-18 months, the team felt more confident in their patient assessment ability and decision making. Confidence in the team grew in the ED through a combination of rapid accessibility, repeated positive interactions and regular management meetings at consultant level. The team now provide regular formal teaching to the ED junior doctors for topics such as chest pain and syncope. The success of the team is due to a combination of the specialist nurses' long experience in cardiology and their continual commitment to improving both patient care and their own knowledge.

The decision to require a prolonged cardiology course over a typical ANP-type master's course was a point of deliberation for us and we gave it careful consideration. The aim of the role was to give an SpR equivalent cardiology opinion to the emergency portals. The basic health assessment equips the individual with the ability to take a history and reach some conclusions when coupled with physical examination findings. The cardiology specific educational programme then ensures that the practitioner is giving an expert and credentialed cardiology opinion. Prescribing is only done on completion of the postgraduate diploma and allows the practitioner to combine clinical reasoning with their expert knowledge of the underpinning trials and evidence base of treatments to make holistic decisions for patients on an individual basis rather than just following a 'one size fits all' protocol. A traditional MSc is generic and would not have afforded the team sufficient specific and credible educational training to offer the expert opinion. The Royal College of Nursing recognises this in its credentialing programme, and the practitioners can have the credential of advanced nurse practitioners with MSc in Cardiology coupled with the Health Assessment and Prescribing modules.

The cardiac assessment nurse team has made a positive impact on the junior doctors. At our centre, junior doctors rotate on a 4-monthly rotation and the nurse-led team is permanent. The team has also expanded to include an advanced nurse practitioner based on the ward working with the junior doctor team to support learning and decision making in real time. Our team's presence on the ward enables back-fill time for junior colleagues to facilitate supernumerary education time for junior doctors to attend timetabled teaching sessions such as journal club. The feedback from junior doctors on their placement in cardiology has shown significantly increased satisfaction as a result of the presence of cardiac assessment nurse team.

A difficulty relating to estimates of the cost of the nurse-led cardiology team is the cost associated with consultant input. This service is not a triage service as nurses work autonomously assessing, forming a working diagnosis, arranging investigations and making treatment plans for inpatient and outpatient care. However, there are situations where nurses require advice from consultants regarding patient care and 1:1 time for nurses to speak to consultants. While we were not able to estimate a cost figure for this time, it was frequently done on the cardiac assessment nurse's 'own' time thereby offsetting cost.

One of the limitations of the current evaluation is the lack of data from SpR-led services prior to service implementation. We are aware that the burden of responsibility of the cardiology $\mathrm{SpR}$ was significant prior to the development of the service. The on-call responsibility meant that the registrar may have to do procedures or manage patients overnight while still carrying on day responsibilities such as clinics and catheter laboratory sessions. There was no limit to the number of referrals for the SpR and there were cases where it was impossible to safely manage acutely ill patients while ensuring referrals were seen within 30 minutes of referral. The new service was a welcomed addition to alleviate the burden of on-call cardiology services as, prior to the new service, the registrar would attend the procedure and the referrals would build up. The increase in the number of experienced members on the on-call team available enabled the nurses to carry on reviewing patients if the SpR needed to assist in the catheter laboratory. There were also benefits from a consultant's perspective as they could be reassured that there was an experienced team in the hospital at all times in the case of a junior registrar.

Another potential issue is the generalisability of our findings. As our hospital is a tertiary specialist unit, we have a high number of cardiology referrals from the community and neighbouring hospitals providing sufficient numbers of patients that require specialist cardiac opinion. It is possible that smaller district general 
hospitals may not have enough patients to require 24 hours a day, 7 days a week cardiology nurse specialist team. We believe that our findings are important because the innovative service we have created may be tailored to meet the demands on hospitals. For hospitals where there are more active consultant-, SpR- or junior doctor-led reviews, a cardiac assessment nursing team could be developed to provide more of an outpatient service.

\section{Conclusion}

We describe our innovative cardiac assessment nurse team that provides a 24 -hour presence in the ED. Supported by the on-call cardiology registrar and consultants, these dedicated nurses can optimise patient management including admission to the cardiology ward or safely discharged with relevant follow-up. However, they also have an integral role in provision of specialist clinic services including fast track palpitations or rapid access chest pain clinics. Furthermore, they provide a managerial role supporting junior doctors in clinical practice, rota coordinating and organising teaching rotas for junior doctors and allied medical professionals. In the current economic climate, saving is of paramount importance. This service provides an estimated saving of $£ 400,000$ each year from bed-days and contributes $£ 121,792$ income for the directorate from outpatient clinic services.

\section{References}

1 British Heart Foundation. UK Factsheet. BHF, 2019. www.bhf.org. uk/research/heart-statistics

2 Bhatnagar P, Wickramasinghe K, Williams ], Rayner M, Townsend $\mathrm{N}$. The epidemiology of cardiovascular disease in the UK 2014. Heart 2015;101:1182-9.
3 O'Neill L, Smith K, Currie P, Elder D, Wei L, Lang C. Nurse-led Early Triage (NET) study of chest pain patients: a long term evaluation study of a service development aimed at improving the management of patients with non-ST-elevation acute coronary syndromes. Eur ] Cardiovasc Nurs 2014;13:253-60.

4 Motherwell DW, Rogers ], Kellagher $\mathrm{M}$ et al. The introduction of a chest pain nurse and fast-track troponin service reduces length of stay of patients presenting with chest pain. Scot Med J 2007;52:6-9.

5 Bonzi M, Fiorelli EM, Angaroni L et al. Predictive accuracy of triage nurses evaluation in risk stratification of syncope in the emergency department. Emerg Med J 2014;31:877-81.

6 Arslanian-Engoren C. Explicating nurses' cardiac triage decisions. J Cardiovasc Nursing 2009;24:50-7.

7 Blue L, Lang E, McMurray JJV et al. Randomised controlled trial of a specialist nurse intervention in heart failure. $B M J$ 2001;323:715-8.

8 Stewart S, Marley JE, Horowitz JD. Effects of a multidisciplinary, home-based intervention on unplanned readmissions and survival among patients with chronic congestive heart failure: a randomised controlled study. Lancet 1999;354:1077-83.

9 Stewart S, MacIntyre K, Hole DJ, Capewell S, McMurray J]. More 'malignant' than cancer? Five-year survival following a first admission for heart failure. Eur ] Heart Fail 2001;3:315-22.

10 David D, Britting L, Dalton J. Cardiac acute care nurse practitioner and 30-day readmission. J Cardiovasc Nurs 2015;30:248-55.

11 Debney MT, Fox KF. Rapid access cardiology - a nine year review. QJM 2012;105:231-4.

Address for correspondence: Dr Chun Shing Kwok, The Heart Centre, Royal Stoke University Hospital, Newcastle Road, Stoke-on-Trent ST4 6QG, UK Email: shingkwok@doctors.org.uk 